\title{
Understanding sarcomas and other rare tumors: an interview with Robin L Jones
}

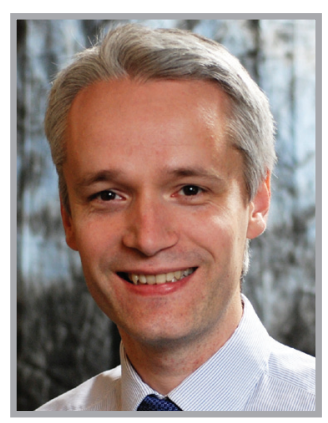

Robin L Jones* speaks to Jade Parker, Commissioning Editor: Robin Jones is a medical oncologist specializing in the treatment of bone and soft tissue sarcomas and Head of the Sarcoma Unit at The Royal Marsden. He completed his medical training at Guy's and St Thomas' Hospital, and his oncology training at The Royal Marsden. His postgraduate research degree, with Professor Dowsett at the Institute of Cancer Research (ICR), evaluated potential predictive and prognostic factors in breast cancer patients treated with neoadjuvant chemotherapy. In January 2010 he was appointed Associate Professor and Director of the Sarcoma Program at the University of Washington and Fred Hutchinson Cancer Research Center in Seattle. He led a successful, grant funded program and continued his translational and clinical trial-based research. The laboratory work with Dr Seth Pollack evaluated novel immunotherapeutic targets in bone and soft tissue sarcomas, and has led to a number of early-phase immunotherapeutic clinical trials in sarcoma. He returned to The Royal Marsden and Institute of Cancer Research in December 2014, as Sarcoma Clinical Trials Team Leader and Consultant Medical Oncologist. He has experience in conducting Phase I, II and III trials, as well as translational studies in sarcoma. He is continuing a number of trials of investigational agents as well as laboratory-based immunotherapy studies.

Accepted for publication: 25 October 2016; Published online: 7 December 2016

Q Can you tell us about your career? What led you to working in sarcoma research?

I trained at Guy's and St Thomas' Hospitals in London and did an intercalated BSc (first class) in medical physics/ radiological sciences. My oncology training was at the Royal Marsden Hospital in London. My postgraduate research thesis evaluated a number of putative predictive and prognostic markers in breast cancer patients treated with neoadjuvant systemic therapy with Professors Ian Smith and Mitch Dowsett. In 2010, I took up a faculty post as head of the sarcoma program at the University of Washington/ Fred Hutchinson Cancer Research Center in Seattle. I initiated a number of Phase I and II clinical trials as well as studies evaluating novel immunotherapeutic therapies in sarcomas, particularly synovial and myxoid liposarcoma. In 2015, I became head of the sarcoma program at the Royal Marsden in London.

\section{Q What would you note as your greatest professional achievement to date? How have you seen the field develop over your career?}

Developing a research interest in sarcomas and setting up a sarcoma research program in Seattle have been professionally gratifying. I first became interested in sarcomas as a trainee at the Royal Marsden Hospital and performed some database studies in liposarcoma [1,2]. I also developed an interest in Phase I clinical trials and other treatment modalities

\section{KEYWORDS}

- clinical trials • rare cancers

- sarcomas 
in sarcomas [3,4]. At the Fred Hutch Cancer Research Center and University of Washington, my colleague Seth Pollack and I performed a number of preclinical and early clinical studies evaluating potential immunotherapeutic targets in sarcomas [5-7]. I also worked with Janet Eary and Eve Rodler on a preoperative trial of the antiangiogenic agent, pazopanib, in localized soft tissue sarcomas. I was an investigator on a number of important Phase III trials in sarcomas, including a trial of trabectedin versus dacarbazine in lipo- and leiomyosarcoma, eribulin versus dacarbazine in lipo- and leiomyosarcomas, and doxorubicin with or without evofosfamide in soft tissue sarcomas [8]. These trials led to the approval of trabectedin and eribulin by the US FDA. I am also the European lead investigator for a Phase III trial of doxorubicin with either a monoclonal antibody to PDGFR- $\alpha$ or placebo, following a promising Phase II trial [9]. I have continued to work on investigator initiated trials and have a number of immunotherapeutic trials due to open. In 2017, I will be the American Society of Clinical Oncology (ASCO) Education Committee lead for sarcoma. I am a member of the Sarcoma Alliance for Research through Collaboration Concept (SARC) Review Committee as well as a member of the American Joint Commission on Cancer (AJCC) soft tissue sarcoma staging Committee. In my post in Seattle, I led a change in the structure of the clinic, which resulted in an increase in referrals and improved patient satisfaction. I have continued to supervise a number of research students in my new post at the Royal Marsden Hospital and I continue to lecture nationally and internationally on soft tissue and bone sarcomas.

\section{Q Soft tissue sarcomas is an umbrella term for an array of various rare cancers including liposarcoma. Can you tell our readers more about liposarcoma in particular \& why it is considered rare?}

Sarcomas account for about $1 \%$ of all adult tumors and are made up of over 80 different histological subtypes. Liposarcomas are one of the most common of these subtypes. However, liposarcomas are also a group of diseases, each with their own unique biology and response to systemic therapy. The most common are well-/de-differentiated liposarcoma, which are characterized by amplifications in CDK4 and $M D M 2$. These tend to present as large primary tumors and local control can be a major challenge. The second group is myxoid liposarcoma characterized in the majority of cases by a specific chromosomal translocation $\mathrm{t}(12 ; 16)$. These tumors tend to present with lower limb primaries and metastasize to unusual locations for sarcomas, such as pleural, pericardial and bone. The third subtype, pleomorphic, is very rare and these tumors have a complex karyotype, with many genetic gains and losses. They tend to have an aggressive clinical course.

Q What is being done to raise awareness of this rare disease?

Many patient groups in conjunction with sarcoma specialists are trying to raise awareness of these rare and complex diseases. As a collective group, all rare cancers account for approximately $25 \%$ of cancers but the outcomes for patients with rare cancers are worse than those for patients with more common cancers. There is a need for more research and more research funding for rare cancers, including sarcomas. The Royal Marsden Sarcoma Unit regularly holds educational events for general practitioners, surgeons, oncologists, other medical professionals and patients regarding sarcomas, outlining their diagnosis, management and advances in treatment.

Q What challenges do physicians face in the diagnosis \& treatment of liposarcoma?

The first issue is that because sarcomas are so rare it can be difficult to establish the diagnosis. Most general practitioners will only see two to three sarcoma patients in their career. Consequently, there can be a delay in the diagnosis of sarcomas. In addition, because there are so many subtypes, the histological diagnosis can also be challenging. An experienced multidisciplinary team should treat sarcoma patients. Due to the rarity, heterogeneity and widespread anatomic primary sites these tumors are very challenging to treat.

Q You are very involved in clinical trials which look at novel agents for treating liposarcoma. How have agents such as eribulin helped overcome the challenges faced by physicians treating the disease?

It is important to increase our knowledge of sarcomas, and clinical trials are very important in increasing our understanding but also developing new effective therapies for patients. 
Due to the biological heterogeneity and rarity of sarcomas it has been difficult to conduct large clinical trials, and consequently few drugs or schedules have been shown to be effective. However, in some subtypes, particularly pediatric cancers such as osteosarcoma, Ewing and rhabdomyosarcoma, systemic therapy has had a huge impact on survival for patients with localized disease. More recently, the tyrosine kinase inhibitor, imatinib, has demonstrated efficacy as post-operative therapy in gastrointestinal stromal tumor. The trials in other sarcoma subtypes have been inconclusive, largely due to the inclusion of heterogeneous subtypes, suboptimal chemotherapy schedules and small patient numbers. However, a recent randomized trial of conventional neoadjuvant epirubicin and ifosfamide has shown a statistically significant benefit in terms of recurrence-free and overall survival compared with histology-tailored chemotherapy [10].

In the metastatic setting, doxorubicin has been the backbone of palliative therapy for 40 years. Recently, a number of drugs have been approved for metastatic disease including pazopanib, trabectedin and eribulin. The Phase III trial randomizing liposarcoma and leiomyosarcoma patients to receive dacarbazine or eribulin should be a survival benefit for eribulin. This is the first Phase III trial in soft tissue sarcomas to demonstrate an overall survival benefit.

\section{Q There is currently some debate into the} clinical trial end points \& their suitability in drug trials, particularly in terms of overall survival versus progression-free survival \& quality of life as a measure of assessing clinical benefit. What are your thoughts on these end points, \& which is most suited for use in sarcoma trials?

Currently, there is no perfect end point for trials in sarcomas. Overall survival is a composite end point and can be influenced by therapies following the treatment being evaluated. In addition, the fact that some sarcoma subtypes can be relatively indolent (with prolonged survival) and others can be very aggressive mean that this is a challenging end point to gauge precisely in the context of an 'all comer' sarcoma trial. Progression-free survival is commonly used as an end point but can also be influenced by the timing of scans and does not take into account the toxicity of therapy. Following on from these considerations, in indolent subtypes prolonged progression-free survival can be a result of the natural biology of the disease rather than direct drug effect. Randomized discontinuation trial design takes into account of this indolent biology. In such a trial, all patients receive a treatment for a length of time prior to the first restaging scan. Patients with disease responding to therapy continue with the same treatment and patients with progressive disease come off trial. Patients with stable disease are randomized to receive either drug or placebo. At subsequent re-staging, patients on placebo can then be recommenced on treatment. Quality of life measures are difficult to formulate for such a heterogeneous group of diseases. In addition, quality of life is such an individual measure that can be difficult to formulize into a set of criteria. There are a number of quality of life measures in sarcoma, but they have been developed mainly in the orthopedic arena for patients with localized disease. More work is required to develop reproducible quality of life measures in metastatic sarcomas.

\section{Q Can you tell our readers about some of} your current research \& other 'hot topics' in the sarcoma field?

My current research focuses on the immunology of various sarcoma subtypes and we have a number of immunotherapy trials being in development. I am continuing to collaborate with my colleagues, Seth Pollack, Alex Lee and Paul Huang on a number of immunotherapy trials as well as laboratory work in sarcomas. I am also running a range of Phase I, II and III trials in various sarcoma subtypes in collaboration with Winette van der Graaf, Charlotte Benson, Aisha Miah, Shane Zaidi, Khin Thway, Cyril Fisher and the Royal Marsden Sarcoma Unit. The results of the recently presented trial of neoadjuvant chemotherapy are also very exciting.

Q How do you see the field evolving over the next 10 years?

The management of sarcomas is likely to evolve greatly over the next few years to involve more emphasis on the underlying biology, which hopefully will guide treatment rather than a generic 'one-size-fits-all' approach to systemic therapy and clinical trial design. The advances that have been made in the diagnosis and management of gastrointestinal stromal tumors clearly show the progress that can be made in 
sarcomas, with three drugs approved for the treatment of metastatic disease and imatinib approved as adjuvant therapy. In addition, it is hoped that the diagnosis of sarcomas will be easier with greater use of novel techniques.

\section{Disclaimer}

The opinions expressed in this interview are those of the interviewee and do not necessarily reflect the views of Future Medicine Ltd.
Financial \& competing interests disclosure RL Jones has been a consultant for Eisai, Lilly, Merck, JJ, Pharmamar, Pfizer, Daiichi, Immodulon, Immunedesign, Adaptimmune. RL Jones has no other relevant affliations or financial involvement with any organization or entity with a financial interest in or financial conflict with the subject matter or materials discussed in the manuscript apart from those disclosed.

No writing assistance was utilized in the production of this manuscript.

\section{References}

1 Jones RL, Fisher C, Al-Muderis O, Judson IR. Differential sensitivity of liposarcoma subtypes to chemotherapy. Eur. J. Cancer 41(18), 2853-2860 (2005).

2 Grosso F, Jones RL, Demetri GD et al. Efficacy of trabectedin (ecteinascidin-743) in advanced pretreated myxoid liposarcomas: a retrospective study. Lancet Oncol. 8(7), 595-602 (2007).

3 Jones RL, Olmos D, Thway K et al. Clinical benefit of Phase I participation for advanced sarcoma patients. Cancer Chemother. Pharmacol. 68(2), 423-429 (2011).

4 Jones RL, McCall J, Adam A et al. Radiofrequency ablation is a feasible therapeutic option in the multi modality management of sarcoma. Eur. J. Surg. Oncol. 36(5), 477-482 (2010).
5 Pollack SM, Jones RL, Farrar EA et al. Tetramer guided, cell sorter assisted production of clinical grade autologous NY-ESO-1 specific CD8 ${ }^{+} \mathrm{T}$ cells. J. Immunother. Cancer 2(1), 36 (2014).

6 Pollack SM, Li Y, Blaisdell MJ et al. NYESO-1/LAGE-1s and PRAME are targets for antigen specific $T$ cells in chondrosarcoma following treatment with 5-Aza-2deoxycitabine. PLoS ONE 7(2), e32165 (2012).

7 Pollack SM, Jungbluth AA, Hoch BL et al. NY-ESO-1 is a ubiquitous immunotherapeutic target antigen for patients with myxoid/round cell liposarcoma. Cancer 118 (18), 4564-4570 (2012).

8 Demetri GD, von Mehren M, Jones RL et al. Efficacy and safety of trabectedin or dacarbazine for metastatic liposarcoma or leiomyosarcoma after failure of conventional chemotherapy: results of a Phase III randomized multicenter clinical trial. J. Clin. Oncol. 34(8), 786-793 (2016).

9 Tap WD, Jones RL, Van Tine BA et al. Olaratumab and doxorubicinversus doxorubicin alone for treatment ofsoft-tissue sarcoma: an open-label Phase $1 \mathrm{~b}$ and randomised Phase 2 trial. Lancet 388(10043), 488-497 (2016)

10 Gronchi A, Ferrari S, Quagliuolo V et al. Full-dose neoadjuvant anthracycline + ifosfamide chemotherapy isassociated with a relapse free survival (RFS) and overall survival (OS) benefitin localized high-risk adult soft tissue sarcomas (STS) of the extremities andtrunk wall: interim analysis of a prospective randomized trial. Presented at: European Society of Medical Oncology Annual Meeting. Copenhagen, Denmark, 7-11 October 2016. 